\title{
Efficacy and predictors of response to inhaled corticosteroid treatment for chronic cough
}

\author{
Ji Young Hong ${ }^{1,2}$, Joo-Hee Kim ${ }^{2,3}$, Sunghoon Park ${ }^{2,3}$, Yong Il Hwang ${ }^{2,3}$, Ki-Suck Jung ${ }^{2,3}$, and \\ Seung Hun Jang ${ }^{2,3}$
}

\begin{abstract}
${ }^{1}$ Division of Pulmonary and Critical Care Medicine, Department of Medicine, Hallym University Chuncheon Sacred Heart Hospital, Chuncheon; ${ }^{2}$ Lung Research Institute of Hallym University College of Medicine, Chuncheon; ${ }^{3}$ Division of Pulmonary, Allergy and Critical Care Medicine, Department of Medicine, Hallym University Sacred Heart Hospital, Anyang, Korea
\end{abstract}

Received: August 24, 2017

Revised : December 21, 2017

Accepted: May 4, 2018

\section{Correspondence to}

\section{Seung Hun Jang, M.D.}

Division of Pulmonary, Allergy and Critical Care Medicine,

Department of Medicine,

Hallym University Sacred Heart

Hospital, 22 Gwanpyeong-ro

17obeon-gil, Dongan-gu,

Anyang 14068, Korea

Tel: $+82-31-380-3718$

Fax: $+82-31-380-3973$

E-mail: chestor@hallym.or.kr
Background/Aims: Inhaled corticosteroids (ICS) are a treatment of choice for eosinophilic airway diseases, but their efficacy for other causes of chronic cough is controversial.

Methods: We conducted a prospective observational study to determine the ICS efficacy and clinical predictors of response to ICS in patients with upper airway cough syndrome (UACS) or unexplained chronic cough (UCC). Sixty-eight patients with UACS and 33 patients with UCC (duration of cough $\geq 8$ weeks) were treated with ICS: $250 \mu \mathrm{g}$ of fluticasone propionate or $400 \mu \mathrm{g}$ of budesonide twice a day at physician's discretion. They were followed after 2 weeks to assess persistent cough which was measured as $\% \%$ to $100 \%$ compared with baseline cough frequency.

Results: The median grade of persistent cough after 2-week ICS treatment was $40 \%$ (interquartile range [IQR], 10 to 70 ) in UACS and was 50\% (IQR, 20 to 70 ) in UCC. The only adverse event was infrequent, mild hoarse voice (five UACS and one UCC). Long duration of cough ( $\geq 52$ weeks) and cough not aggravated by cold air exposure were predictors of a poorer response to short course ICS treatment (logistic regression analysis, $p=0.018$ and $p=0.031$, respectively). However, prolonged treatment with ICS more than 2 weeks was more effective in patients with long cough duration ( $\geq 52$ weeks).

Conclusions: Short course ICS treatment has modest efficacy on UACS and UCC without significant adverse events. Duration of cough and cough triggered by cold air exposure were the clinical factors associated with ICS response. Extended treatment with ICS may be beneficial in patients with long duration of cough.

Keywords: Cough; Adrenal cortex hormones; Treatment outcome

\section{INTRODUCTION}

Chronic cough lasting for more 8 weeks is a common presenting symptom in clinical practice [1]. Chronic cough has a reported prevalence between $14 \%$ and $23 \%$ [2]. Persistent cough is associated with impaired quality of life and greater medical care utilization $[3,4]$. Irwin et al. [5] reported that a cause for chronic cough could be diagnosed in up to $98 \%$ using an 'anatomic diagnostic protocol.' In patients with chronic cough with a normal chest radiograph, almost all identified causes are upper airway cough syndrome (UACS), gastroesophageal reflux disease (GERD), and cough variant asthma (CVA) $[5,6]$.

The diagnostic trial approach identifies the cough etiology by confirming that the cough responds to a trial of therapy that is specific for the suspected cause [7]. 
McGarvey et al. [6] showed that resolution of symptoms occurs after diagnosis-specific treatment that includes inhaled corticosteroids (ICS) for CVA, antihistamine/ decongestant for UACS and proton pump inhibitor for GERD.

However, several studies reported a high prevalence of unexplained chronic cough (UCC) that persists despite appropriate investigation and treatment $[8,9]$. In addition, some studies showed that the response to treatment trials provides little information about the etiology of the cough [10]. A systematic review reported that the effect of proton pump inhibitor on cough associated with GERD is less universal than suggested in consensus guidelines [10]. Tanaka et al. [11] reported that loratadine ( $\mathrm{H} 1$ antihistamine) reduced the number of coughs both in patients with UCC and in patients with nasal disease. Contrary to common belief [12], studies report that ICS reduces non-asthmatic chronic cough $[13,14]$. It is hypothesized that epithelial damage due to airway inflammation in non-asthmatic chronic cough could lead to heightened cough-reflex sensitivity $[15,16]$. This pathophysiological mechanism may explain the effects of ICS. However, clinical evidence to support the use of ICS for non-asthmatic chronic cough is insufficient. Moreover, corticosteroid responsive factors the optimal duration or doses of ICS treatment for patients with chronic cough remain to be determined.

The aims of this study are to investigate the efficacy of short course ICS treatment and to identify clinical predictors of the response to ICS in patients with UACS or UCC.

\section{METHODS}

\section{Patients recruitment}

Subjects were recruited between June 2009 and December 2015 at Hallym University Sacred Heart Hospital, a tertiary referral hospital in South Korea. Inclusion criteria were: (1) older than 18 years, chronic cough longer than 8 weeks; (2) normal chest X-ray; (3) no evidence of asthma as indicated by normal metacholine airway responsiveness (a provocation concentration of methacholine $8 \mathrm{mg} / \mathrm{mL}$ failed to cause a fall in forced expiratory volume per 1 second $\left[\mathrm{FEV}_{1}\right]$ of $20 \%\left[\mathrm{PC}_{2 \mathrm{O}}\right]$ or more); and (4) no prior experience of wheezing. Exclusion cri- teria were: (1) symptoms suggestive of respiratory infection, such as fever, sore throat, or suppurative nasal discharge in the preceding 8 weeks; (2) use of other drugs that account for the cough, such as angiotensin-converting enzyme inhibitors; (3) current treatment that might influence the cough status within the 5 days before enrollment; (4) frequent gastroesophageal reflux symptoms; (5) inability to produce sputum despite sputum induction with hypertonic saline; and (6) sputum eosinophil count $\geq 3 \%$. The protocol was approved by the Ethics Review Committee (IRB number: 2009-Ioo6) and written informed consent was obtained from all patients.

\section{Patient evaluations and clinical measurements}

All patients underwent an evaluation that included history and physical examination, spirometry, methacholine bronchial provocation test (MBPT), chest X-ray, paranasal sinus (PNS) X-ray and sputum examination for eosinophil count. Bronchial challenge testing with methacholine was performed using the technique of Chai et al. [17]. Concentrated methacholine solution, from 0.075 to $25 \mathrm{mg} / \mathrm{mL}$, was inhaled five times through a nebulizer, and $\mathrm{FEV}_{1}$ was measured after each inhalation. All participants had normal methacholine airway responsiveness because subjects with a $\mathrm{PC}_{20} \mathrm{FEV}_{1}$ value of $8 \mathrm{mg} / \mathrm{mL}$ or less were excluded.

Sputum was induced with $3 \%$ hypertonic saline in the few patients who were unable to expectorate, as previous described [18]. Sputum cells were examined by simple direct smear with Wright stain to determine differential cell count. The cough characteristics were recorded using questionnaires. Cough frequency was graded as: (1) infrequently in a day; (2) frequently less than hourly; (3) hourly; and (4) constant, nearly all day. Cough severity was graded as: (1) no effect on daily living; (2) mild effect on daily living; (3) marked effect on daily living; and (4) unable to work due to severe cough.

Patients who met the eligible criteria were enrolled and treated with inhaled fluticasone propionate $250 \mu \mathrm{g}$ twice a day by diskus device or budesonide $400 \mu \mathrm{g}$ twice a day by turbuhaler device at the physician's discretion for 2 weeks. All patients who were followed after this 2-week ICS treatment and agreed to additional treatment were considered for additional ICS treatment. Forty-two patients were treated with ICS for longer than 2 weeks. 
T1 was defined as the period after 2 weeks of ICS treatment and $\mathrm{T} 2$ was defined as the period after additional ICS treatment. The patients were followed at the outpatient clinic after treatment with ICS (at T1 in all patients and at $\mathrm{T} 2$ in 42 patients with additional treatment).

The primary outcome measurement was the degree of persistent cough after 2-week treatment, and the secondary outcome measurements were drug compliance and adverse events. The degree of persistent cough was graded by the subject as $\% \%$ to $100 \%$. Zero percent meant no persistent cough. If any cough was present, its degree of frequency was graded by the subject by comparing the post-ICS cough with the baseline cough frequency, which was assigned a grade of $100 \%$.

Patients were categorized UACS or UCC according to the following definitions. UACS was defined as having the following features: PNS mucosal thickening on PNS $\mathrm{X}$-ray or more than two rhinitis symptoms among rhinorrhea, sneezing, stuffiness, itching or medical history of allergic rhinitis or sense of post-nasal drip. The patients with UCC were defined as those without the characteristics of UACS.

A poor response to 2-week ICS treatment was defined as having a persistent cough with a grade of $>50 \%$ of baseline cough frequency (100\%) after treatment.

\section{Statistical analysis}

Categorical variables were analyzed using the Fisher's exact test and continuous variables using Mann-Whitney test. Multivariate logistic regression analysis was performed to evaluate potential clinical predictors for poor response to short course ICS treatment in patients with UACS or UCC. The non-parametric Friedman test with Dunn's posttest was used to evaluate longitudinal changes in the degree of remnant cough percentages after treatment with ICS.

All statistical analyses were conducted using PASW Statistics version 20 (IBM Co., Armonk, NY, USA) and GraphPad Prism version 5.0 (GraphPad, San Diego, CA, USA).

\section{RESULTS}

\section{Baseline characteristics}

The recruitment period was from June 2009 to Decem- ber 2015. Among 132 patients (90 patients with UACS, 42 patients with UCC) who met all the eligible criteria, 101 patients $(76.5 \%)$ were successfully followed (median length of follow-up was 18.0 days [interquartile range (IQR), 14 to 41.5]). Sixty-eight patients with UACS and thirty-three patients with UCC were successfully followed. The pattern of clinical features among the UACS group was PNS mucosal thickening on X-ray $(n=19)$, rhinitis symptoms $(n=29)$, medical history of allergic rhinitis $(\mathrm{n}=29)$, and sense of post-nasal drip ( $\mathrm{n}=31)$; $49 \%$ had a single feature, $44 \%$ had two features, and $7 \%$ had three features.

Baseline characteristics of the patients are shown in Table 1. No significant differences were found between the UACS and UCC groups. Both groups had greater than $90 \%$ drug compliance. Forty-two patients (41.6\%) continued the ICS treatment longer than 2 weeks (median treatment duration, 42 days [IQR, 28.8 to 44.0 ]).

\section{Response to inhaled corticosteroids}

The median grade of persistent cough frequency after 2-week ICS treatment was $40 \%$ (IQR, 10 to 70 ) in patients with UACS and 50\% (IQR, 20 to 70) in patients with UCC. The degree of persistent cough after 2-week ICS treatment did not differ significantly between the UACS and UCC groups (Mann-Whitney test, $p=0.649$ ). A good response to 2-week ICS treatment, a reduction in persistent cough grade by more than $50 \%$, was reported by $67.3 \%$ of all patients. This good response was reported by $63.6 \%$ of patients with UACS and $67.6 \%$ of patients with UCC (Fig. 1). Persistent cough after 2-week ICS treatment showed a significant trend to increase as past duration of cough, reported as baseline, increased (linear trend analysis, $p=0.004$ ) (Fig. 2). The median persistent cough grade after 2-week ICS treatment was significantly higher in patients whose duration of cough was $\geq 52$ weeks than in patients whose duration of cough was $\leq 12$ weeks (cough duration $\geq 52$ weeks: $65 \%$ grade vs. 30 for cough duration $\leq 12$ weeks: Dunn's multiple comparison test, $p<0.05$ ).

\section{Predictors of inhaled corticosteroid responsiveness}

ICS response and clinical factors such as cause of cough, cough aggravation by cold air exposure, nocturnal awakening by cough, duration of cough, smoking status, sex, baseline cough frequency and severity grade 
Table 1. Characteristics of study participants by cough category

\begin{tabular}{|c|c|c|c|}
\hline Variable & Total $(\mathrm{n}=101)$ & UACS $(\mathrm{n}=68)$ & $\mathrm{UCC}(\mathrm{n}=33)$ \\
\hline Age, yr & $39(30.5-48.5)$ & $37(30.3-45.0)$ & $47(30-55 \cdot 5)$ \\
\hline Male sex & $35(34.7)$ & $25(36.8)$ & $10(30.3)$ \\
\hline $\mathrm{BMI}, \mathrm{kg} / \mathrm{m}^{2}$ & $22.4(20.3-25.3)$ & $22.4(20.3-25.4)$ & $22.7(20.3-23.8)$ \\
\hline \multicolumn{4}{|l|}{ The frequency of cough } \\
\hline Infrequently in a day & $28(27.7)$ & $16(23 \cdot 5)$ & $12(36.4)$ \\
\hline Frequently, less than hourly & $44(43.6)$ & $28(41.2)$ & $16(48.5)$ \\
\hline Hourly & $26(25 \cdot 7)$ & $22(32.4)$ & $4(12.1)$ \\
\hline Constant, nearly all day & $3(3.0)$ & $2(2.9)$ & $1(3.0)$ \\
\hline \multicolumn{4}{|l|}{ The severity of cough } \\
\hline No effect on daily living & $36(35 \cdot 6)$ & $21(30.9)$ & $15(45 \cdot 5)$ \\
\hline Mild effect on daily living & $43(42.6)$ & $29(42.6)$ & $14(42.4)$ \\
\hline Marked effect on daily living & $21(20.8)$ & $17(25)$ & $4(12.1)$ \\
\hline Unable to work due to severe cough & $1(1.0)$ & $1(1.5)$ & ० \\
\hline \multicolumn{4}{|l|}{ The duration of cough, wk } \\
\hline$\geq 8$ and $\leq 12$ & $44(43.6)$ & $30(44.1)$ & $14(42.4)$ \\
\hline$>12$ and $\leq 24$ & $28(27 \cdot 7)$ & $20(29.4)$ & $8(24.2)$ \\
\hline$>24$ and $\leq 51$ & $9(8.9)$ & $5(7 \cdot 4)$ & $4(12.1)$ \\
\hline$\geq 52$ & $20(19.8)$ & $13(19.1)$ & $7(21.2)$ \\
\hline Cough duration, wk & $40(20-75)$ & $50(20-70)$ & $40(12.5-70)$ \\
\hline Cough aggravated by cold air exposure & $75(74 \cdot 3)$ & $53(77 \cdot 9)$ & $22(66.7)$ \\
\hline Current smoker & $11(10.9)$ & $7(10.3)$ & $4(12.1)$ \\
\hline \multicolumn{4}{|l|}{ ICS } \\
\hline Fluticasone diskus & $85(84.2)$ & $55(80.9)$ & $30(90.9)$ \\
\hline Budesonide turbuhaler & $16(15.8)$ & $13(19.1)$ & $3(9.1)$ \\
\hline Drug compliance, \% & $100(92.9-100)$ & $100(92.9-100)$ & $100(90.1-100)$ \\
\hline Persistent cough after 2 wk, $\%$ & $40(20-70)$ & $40(10-70)$ & $50(20-70)$ \\
\hline ICS treatment more than $2 \mathrm{wk}$ & $42(41.6)$ & $28(41.2)$ & $14(42.4)$ \\
\hline Duration of ICS treatment, day & $18(14-41.5)$ & $18(14-40.8)$ & $16(14-42)$ \\
\hline
\end{tabular}

Values are presented as median (interquartile range) or number (\%).

UACS, upper airway cough syndrome; UCC, unexplained chronic cough; BMI, body mass index; ICS, inhaled corticosteroid.

were evaluated (Table 2). The clinical factors associated with greater persistent cough (poor ICS response) (Fisher's exact test, $p<0.1)$ were cough not aggravated by cold air exposure $(p=0.054)$ and duration of cough $\geq 52$ weeks $(p=0.043)$. We conducted a multivariate analysis to identify predictors for poor response to short course ICS treatment indicated as degree of remnant cough more than $50 \%$ of baseline frequency (Table 3). Based on the preceding results and previous studies showing a reduced effect of ICS in smokers [19], we included the variables current smoking, cough aggravated by cold air exposure and duration of cough $\geq 52$ weeks in the multivariate logistic regression analysis. The analysis demonstrated that the duration of cough $\geq 52$ weeks and cough not aggravated by cold air exposure were predicting factors for poor response to short course ICS treatment.

\section{Changes of persistent cough grade after ICS treat- ment longer than 2 weeks}

Fig. 3 shows the longitudinal analysis of persistent cough according to prior duration of cough ( $<52$ weeks 


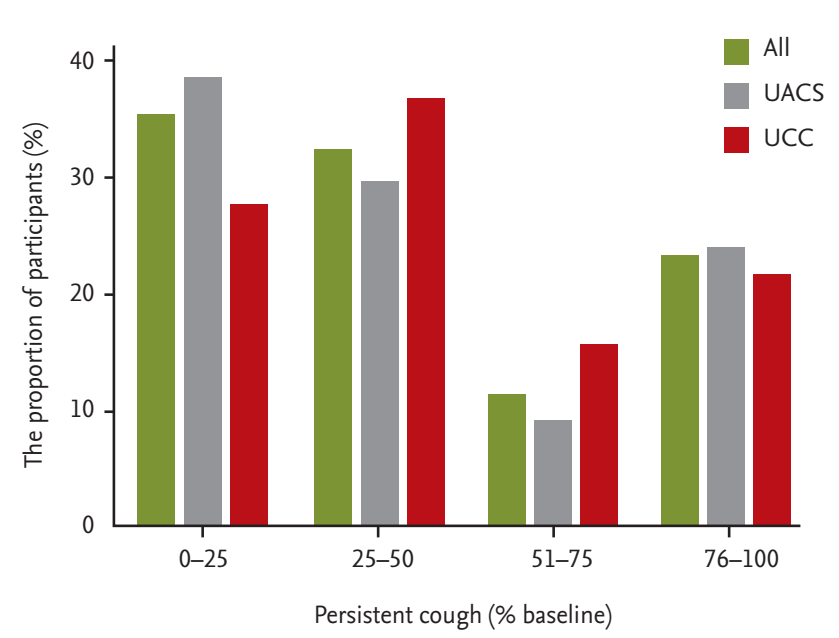

Figure 1. The proportion of study participants according to the degree of persistent cough after 2-week treatment with inhaled corticosteroid. A good response to 2-week inhaled corticosteroid treatment was defined as having a persistent cough after treatment that was $\leq 50 \%$ of baseline cough frequency level (100\%) after treatment. UACS, upper airway cough syndrome; UCC, unexplained chronic cough.

vs. $\geq 52$ weeks) among the 42 study participants who received ICS treatment longer than 2 weeks. This analysis included 14 patients with UCC (66.7\%) and 28 patients with UACS (33.3\%). A good response to short course 2-week ICS treatment was experienced by 28 of these 42 patients $(66.6 \%)$. The median duration of additional ICS treatment was 28 days (IQR, 14 to 28 ). T1 was defined as the period immediately after 2-week ICS treatment, and $\mathrm{T} 2$ was defined as the period after additional ICS treatment. Fig. 3 shows the change of persistent cough, not the good response to ICS. The degree of persistent cough decreased between To (initial) and $\mathrm{T} 1$ (2-week ICS treatment) irrespective of prior duration of cough (Friedman test with Dunn's post-test, $p<0.001$ in both groups). In contrast, the decrease in persistent cough between $\mathrm{T} 1$ (after 2-week ICS treatment) and T2 (after the additional ICS treatment) differed according to the prior duration of cough. The persistent cough significantly decreased between $\mathrm{T} 1$ and $\mathrm{T} 2$ (median persistent cough, T1: 50\%, T2: $0 \%, p<0.05)$ in the subjects whose duration of cough was $\geq 52$ weeks. However, in the subjects whose prior duration of cough was $<52$ weeks, it decreased steadily but not significantly (median persistent cough, T1: 40\%, T2: 10\%, p > 0.05). This result shows that treatment with ICS for more than 2

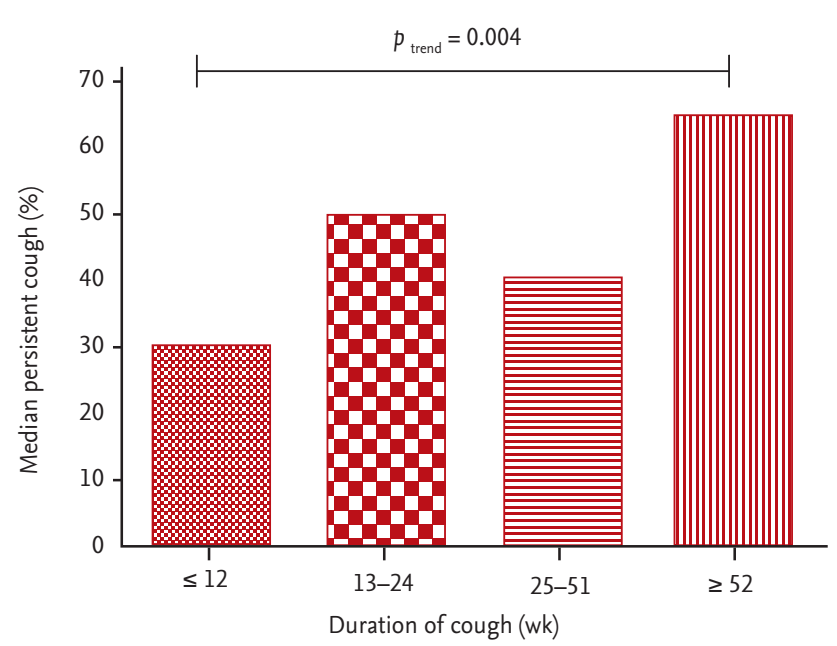

Figure 2. Median degree of persistent cough after 2-week treatment with inhaled corticosteroid varied according to duration of cough reported at baseline $(p=0.004$, linear trend test).

weeks may reduce the remnant cough in patients with long cough duration ( $\geq 52$ weeks).

\section{Local side effects}

Six patients (five UACS and one UCC) reported a mild degree of husky voice after 2 weeks ICS treatment. No other adverse events were reported. No patients discontinued the treatment because of side effects.

\section{DISCUSSION}

Chronic cough is often poorly treated and causes a high level of morbidity in the community [20]. The paucity of data about the management of UCC persists despite substantial investigation [21]. A new perspective beyond 'anatomic diagnostic protocol' is suggested. Explanations of the pathogenesis of UCC have focused on cough reflex hypersensitivity and airway inflammation [9,21]. Pavord and Chung [22] explained that cough depends on combined effects of intrinsic abnormality of cough reflex and aggravating factors. According to this model for the pathogenesis of chronic cough, the treatment directed against potential aggravating factors, such as UACS or GERD, will not achieve satisfactory results if the heightened cough reflex remains untreated. Neuromodulatory agents (amitriptyline, gabapentin, and morphine) and novel antitussive drugs that can po- 
Table 2. The relationship of clinical factors and degree of persistent cough, relative to baseline, after 2-week treatment with inhaled corticosteroid

\begin{tabular}{|c|c|c|c|c|c|c|}
\hline \multirow{2}{*}{ Clinical factor } & \multirow{2}{*}{ Number } & \multicolumn{4}{|c|}{ Degree of persistent cough } & \multirow{2}{*}{$p$ value $^{\mathrm{a}}$} \\
\hline & & $0 \%-25 \%$ & $26 \%-50 \%$ & $51 \%-75 \%$ & $76 \%-100 \%$ & \\
\hline UACS & 33 & $26(38.2)$ & $20(29.4)$ & $6(8.8)$ & $16(23.5)$ & 0.560 \\
\hline UCC & 68 & $9(27 \cdot 3)$ & $12(36.4)$ & $5(15.7)$ & $7(21.2)$ & \\
\hline PNS & 19 & $7(36.8)$ & $5(26.3)$ & $3(15.8)$ & $4(21.1)$ & 0.815 \\
\hline No PNS & 82 & $28(34.1)$ & $27(32.9)$ & $8(9.8)$ & $19(23.2)$ & \\
\hline R/AR/PND & 61 & $23(37 \cdot 7)$ & $20(32.8)$ & $5(8.2)$ & $13(21.3)$ & 0.652 \\
\hline No R/AR/PND & 40 & $12(30)$ & $12(30)$ & $6(15)$ & $10(25)$ & \\
\hline Cough tendency $^{\mathrm{b}}$ & 43 & $15(34.9)$ & $13(30.2)$ & $3(7.0)$ & $12(27 \cdot 9)$ & 0.603 \\
\hline No cough tendency & 58 & $20(34 \cdot 5)$ & $19(32.8)$ & $8(13.8)$ & $11(19.0)$ & \\
\hline Cough aggravated by cold air exposure & 75 & $27(36.0)$ & $28(37 \cdot 3)$ & $6(8)$ & $14(18.7)$ & 0.054 \\
\hline $\begin{array}{l}\text { Cough not aggravated by cold air } \\
\text { exposure }\end{array}$ & 26 & $8(30.8)$ & $4(15 \cdot 4)$ & $5(19.2)$ & $9(34 \cdot 6)$ & \\
\hline Nocturnal awakening by cough & 39 & $13(33 \cdot 3)$ & $12(30.8)$ & $5(12.8)$ & $9(23.1)$ & 0.972 \\
\hline No nocturnal awakening by cough & 62 & $22(35 \cdot 5)$ & $20(32.3)$ & $6(9.7)$ & $14(22.6)$ & \\
\hline Current smoker & 11 & $2(18.2)$ & $4(36.4)$ & $1(9.1)$ & $4(36.4)$ & 0.524 \\
\hline Ex-smoker/non-smoker & 90 & $33(36.7)$ & $28(31.1)$ & $10(11.1)$ & $19(21.1)$ & \\
\hline Female & 66 & $25(37.9)$ & $22(33 \cdot 3)$ & $5(7.6)$ & $14(21.2)$ & 0.414 \\
\hline Male & 35 & $10(28.6)$ & $10(28.6)$ & $6(17 \cdot 1)$ & $9(25 \cdot 7)$ & \\
\hline Duration of cough $<52 \mathrm{wk}$ & 81 & $32(39 \cdot 5)$ & $26(32.1)$ & $9(11.1)$ & $14(17 \cdot 3)$ & 0.043 \\
\hline Duration of cough $\geq 52 \mathrm{wk}$ & 20 & $3(15.0)$ & $6(30.0)$ & $2(10.0)$ & $9(45.0)$ & \\
\hline Frequency of cough & & & & & & 0.955 \\
\hline Infrequently or frequent, less than hourly & 72 & $24(33 \cdot 3)$ & $24(33 \cdot 3)$ & $8(11.1)$ & $16(22.2)$ & \\
\hline Hourly or constant, nearly all day & 29 & $11(37 \cdot 9)$ & $8(27.6)$ & $3(10.3)$ & $7(24.1)$ & \\
\hline Severity of cough & & & & & & 0.931 \\
\hline No or mild effect on daily living & 79 & $26(32.9)$ & $26(32.9)$ & $9(11.4)$ & $18(22.8)$ & \\
\hline $\begin{array}{l}\text { Marked effect on daily living or unable } \\
\text { to work }\end{array}$ & 22 & $9(40.9)$ & $6(27 \cdot 3)$ & $2(9.1)$ & $5(22.7)$ & \\
\hline Fluticasone diskus & 85 & $30(35 \cdot 3)$ & $27(31.8)$ & $11(12.9)$ & $17(20.0)$ & 0.306 \\
\hline Budesonide turbuhaler & 16 & $5(31.2)$ & $5(31.2)$ & o & $6(37.5)$ & \\
\hline
\end{tabular}

Values are presented as number (\%).

UACS, upper airway cough syndrome; UCC, unexplained chronic cough; PNS, paranasal sinus mucosal thickening on X-ray; R, rhinitis defined as two or more among rhinorrhea, sneezing, stuffiness, itching; AR, present or past medical history of allergic rhinitis; PND, sense of post nasal drip.

${ }^{\mathrm{a}}$ Chi-square test.

${ }^{\mathrm{b}}$ Cough tendency: chronic intermittent cough for more than 2 years.

tentially down-regulate cough hypersensitivity (such as transient receptor potential vanilloid-1 [TRPV1] antagonist, selective cannabinoid agonists [CB2 agonists], and $\mathrm{P}_{2} \mathrm{X}_{3}$ receptor antagonists) are being suggested as therapeutic agents $[20,21]$.
Our findings also contrast with the existing 'anatomic diagnostic protocol.' Our study demonstrates that short course ICS treatment has modest efficacy on UACS and UCC. Cough decreased more than 50\% baseline after ICS treatment in $67.6 \%$ of patients with UACS and 
Table 3. The clinical factors associated with poor response (persistent cough $>50 \%$ of baseline cough frequency) after 2 -week treatment with inhaled corticosteroid

\begin{tabular}{|c|c|c|c|c|}
\hline \multirow{2}{*}{ Clinical parameters } & \multicolumn{2}{|c|}{ Univariate } & \multicolumn{2}{|c|}{ Multivariate } \\
\hline & $p$ value & OR $(95 \% \mathrm{CI})$ & $p$ value & OR $(95 \% \mathrm{CI})$ \\
\hline UACS (vs. UCC) & 0.689 & $1.20(0.49-2.86)$ & & \\
\hline PNS (vs. no PNS) & 0.745 & $1.19(0.42-3.36)$ & & \\
\hline R/AR/PND (vs. no R/AR/PND) & 0.277 & $0.63(0.27-1.45)$ & & \\
\hline $\begin{array}{l}\text { Cough not aggravated by cold air exposure } \\
\text { (vs. cough aggravated by cold air exposure) }\end{array}$ & 0.014 & $3.21(1.27-8.10)$ & 0.018 & $3.16(1.22-8.22)$ \\
\hline Nocturnal awakening by cough (vs. no awakening) & 0.706 & $1.18(0.51-2.73)$ & & \\
\hline Current smokers (vs. ex-smokers/non-smokers) & 0.385 & $1.75(0.49-6.22)$ & 0.462 & $1.66(0.43-6.42)$ \\
\hline Female (vs. male) & 0.157 & $0.54(0.23-1.27)$ & & \\
\hline Duration of cough $\geq 52 \mathrm{wk}$ (vs. $<52 \mathrm{wk}$ ) & 0.028 & $3.08(1.13-8.42)$ & 0.031 & $3.16(1.11-8.99)$ \\
\hline \multicolumn{5}{|l|}{ Frequency of cough } \\
\hline $\begin{array}{l}\text { Hourly or constant, nearly all day } \\
\text { (vs. infrequently or frequently, less than hourly) }\end{array}$ & 0.912 & $1.05(0.42-2.61)$ & & \\
\hline \multicolumn{5}{|l|}{ Severity of cough } \\
\hline $\begin{array}{l}\text { Marked effect on daily living or unable to work } \\
\text { (vs. no or mild effect on daily living) }\end{array}$ & 0.836 & $0.99(0.33-2.47)$ & & \\
\hline Budesonide (vs. fluticasone) & 0.724 & $1.22(0.40-3.70)$ & & \\
\hline
\end{tabular}

OR, odds ratio; CI, confidence interval; UACS, upper airway cough syndrome; UCC, unexplained chronic cough; PNS, paranasal sinus mucosal thickening on X-ray; R, rhinitis defined as two or more among rhinorrhea, sneezing, stuffiness, itching; AR, present or past medical history of allergic rhinitis; PND, sense of post nasal drip.
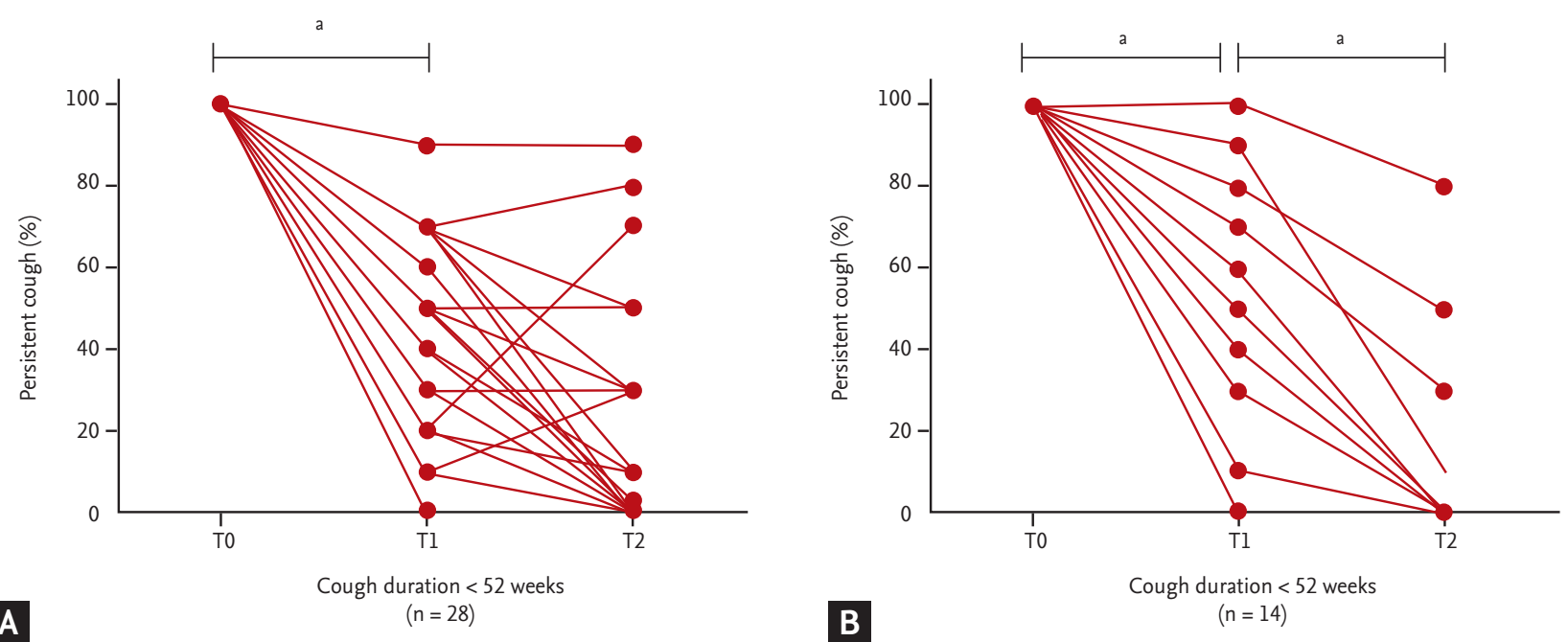

Figure 3. The decrease of persistent cough (\% relative to baseline) after prolonged treatment with inhaled corticosteroid. (A) Patients with cough duration $<52$ weeks $(n=28)$. (B) Patients with cough duration $\geq 52$ weeks $(n=14)$. In patients with cough duration longer than 52 weeks, prolonged treatment with ICS for more than 2 weeks was followed by a significantly lower degree of persistent cough (median persistent cough [Q1 to Q3], T1: 50\% [30 to 80], T2: $0 \%$ [o to 35], $p<0.05$ ). To, baseline; T1, 2 weeks after To; T2, more than 2 weeks after T1. ${ }^{\mathrm{a}} \mathrm{p}<0.05$ analyzed by Friedman test with Dunn's post-test. 
63.6\% patients with UCC (Fig. 1). This result contrasts with the existing recommendation of older generation $\mathrm{H} 1$ antihistamines when UACS is suspected as a cause of cough [23]. Notably, the evidence supporting antihistamines in UACS is not strong and based largely on success rates of antihistamine in patients with chronic cough due to a variety of causes [24]. Only one double-blind placebo-controlled study demonstrated efficacy of antihistamines for the treatment of cough due to UACS [25]. A previous study reported that antihistamine was similarly effective in patients with UCC and patients with nasal disease [11]. Considering that cough suppression mechanism of antihistamines includes inhibiting the airway inflammation and antagonizing the TRPV1 cough receptor [23], a response to antihistamines does not predict an effect specific for UACS, but rather indicates an antitussive effect regardless of cause of cough.

An expert panel report suggests that ICS should not be prescribed in adult patients with UCC and negative tests for asthma [21]. The suggestion is supported by the study of Pizzichini et al. [12] showing no beneficial effect of budesonide in nonasthmatic, noneosinophilic subjects. However, the lack of effect of budesonide in that study may be due to the small number of study participants (23 placebo, 21 budesonide), the inclusion of patients with a longstanding cough (11.8 years in placebo arm and 9.8 years in budesonide arm) and the short duration (2 weeks) of budesonide treatment. However, several studies reported a beneficial response to ICS regardless of the cause of chronic cough, consistent with the present study $[13,14,26]$. Chaudhuri et al. [14] found that cough severity and sputum eosinophilic cationic protein (ECP) levels are modestly reduced by inhaled fluticasone regardless of the cause of the cough. A previous study found a marked, favorable effect of fluticasone in nonsmokers with non-asthmatic cough [13]. Ribeiro et al. [26] showed that high-dose inhaled beclomethasone achieved an excellent response in a subgroup of patients with chronic cough that was not related to airway hyperresponsiveness. Similarly, Han et al. [27] reported that 2-week ICS treatment was as effective as conventional antitussive agents for controlling chronic idiopathic cough.

An intriguing research question is whether optimal duration of ICS for effective treatment varies according to the duration of prior cough. In our study, 2-week ICS treatment was more effective in patients with shorter duration of cough (Fig. 2), and cough duration $\geq 52$ weeks was an independent predictor of poor response to 2-week ICS treatment. Interestingly, prolonging the duration of ICS treatment, in selected patients, was effective in patients with cough duration $\geq 52$ weeks (Fig. 3). To date, the regimen, dosing schedule and treatment duration for ICS therapy of chronic cough have not be standardized. Several previous studies evaluated how chronic cough changed after 2-week ICS treatment $[13,14]$. Boulet et al. [16] showed that 4-week ICS treatment did not improve the response rate compared with the placebo group. A previous study showed that extending treatment to 4 weeks did not improve the response rate compared with 2-week ICS treatment [12]. Further studies of favorable responders to ICS treatment and optimal application are needed.

The mechanism of action of ICS in chronic cough is considered to act through its effect on airway inflammation. Chronic cough is the result of epithelial damage due to inflammatory cell infiltration [16]. Lee et al. [15] reported that a subgroup with chronic nonproductive cough that was associated with airway inflammation due to lymphocyte infiltration improved partially after oral steroid therapy. In contrast, Chaudhuri et al. [14] found that after 2-week ICS treatment, symptom severity and sputum ECP improved but proinflammatory mediators, such as prostaglandin E2 ( $\mathrm{PGE} 2$ ), interleukin 8 (IL-8), myeloperoxidase, and tumor necrosis factor $\alpha$ (TNF- $\alpha$ ), were unaltered in patients with chronic cough of more than 1-year duration. Whether proinflammatory mediators as well as the cough symptoms can be reduced by prolonged ICS treatment needs to be investigated.

The efficacy of ICS on UACS in our study could be interpreted as reducing generalized mucosal inflammation affecting upper and lower airways [28]. In our study, cough not provoked by cold air responded poorly to ICS treatment. UCC provoked by cold air exposure is associated with increased capsaicin cough sensitivity [29]. Based on a new paradigm the "cough hypersensitivity syndrome," blocking or desensitization of TRPV1 (cough receptor) claimed attention [30]. Antonicelli et al. [31] reported that ICS treatment reversed ethanol-induced TRPVı activation. Similarly, ICS may be effective 
in cold air-induced TRPVı activation. Additional research about the mechanism of ICS treatment for chronic cough will help identify the good responders to ICS treatment.

This study had several limitations. First, we did not compare the effect of ICS with a placebo because there was no control group. Because cough tends to improve spontaneously over time, it is necessary to interpret the results carefully in the absence of a control group. Also, the use of two different types of ICS in this study may have affected the outcome. Second, improvement in the cough frequency was measured only by subjective recall by the subject. Cough-reflex testing and direct assessment of airway inflammation to interpret the mechanism, were not conducted. Third, the number of participants who received the extended treatment with ICS for more than 2 weeks was small. Lastly, the diagnosis of UACS and UCC was primarily based on clinical judgment rather than objective tests. Therefore, the possibility that the UACS and UCC groups of this study included other diseases should be considered.

In conclusion, this study shows that short course ICS treatment reduces cough frequency in UACS and UCC without significant adverse events. Long duration of cough $\geq 52$ weeks and cough not aggravated by cold air exposure were clinical predictors strongly associated with poor ICS response. Treatment with ICS longer than two weeks may be effective in patients with cough of long duration. Determining optimal dose and duration of ICS for patients with UACS and UCC needs further study.

\section{KEY MESSAGE}

1. Short course inhaled corticosteroid (ICS) treatment has modest efficacy for upper airway cough syndrome and unexplained chronic cough without significant adverse events.

2. Patients with short-term cough and cough triggered by cold air exposure experienced a relatively better response to ICS.

3. Extended treatment with ICS may be beneficial in patients with long duration of cough.

\section{Conflict of interest}

No potential conflict of interest relevant to this article was reported.

\section{Acknowledgments}

This research was supported by the Bio \& Medical Technology Development Program of the National Research Foundation (NRF) funded by the Korean government (MSIT) (NRF-2017M3A9E8033225). This work was supported by a grant from the National Research Foundation of Korea funded by the Korean government (NRF 2017R1CiB5017879).

\section{REFERENCES}

1. Irwin RS, Madison JM. The diagnosis and treatment of cough. N Engl J Med 2000;343:1715-1721.

2. Wynder EL, Lemon FR, Mantel N. Epidemiology of persistent cough. Am Rev Respir Dis 1965;91:679-700.

3. French CL, Irwin RS, Curley FJ, Krikorian CJ. Impact of chronic cough on quality of life. Arch Intern Med 1998;158:1657-1661.

4. Schappert SM, Rechtsteiner EA. Ambulatory medical care utilization estimates for 2007. Vital Health Stat 13 2011;169:1-38.

5. Irwin RS, Corrao WM, Pratter MR. Chronic persistent cough in the adult: the spectrum and frequency of causes and successful outcome of specific therapy. Am Rev Respir Dis 1981;123:413-417.

6. McGarvey LP, Heaney LG, Lawson JT, et al. Evaluation and outcome of patients with chronic non-productive cough using a comprehensive diagnostic protocol. Tho$\operatorname{rax}$ 1998;53:738-743.

7. McGarvey LP. Cough. 6: Which investigations are most useful in the diagnosis of chronic cough? Thorax 2004; 59:342-346.

8. McGarvey L. The difficult-to-treat, therapy-resistant cough: why are current cough treatments not working and what can we do? Pulm Pharmacol Ther 2013;26:528531.

9. Birring SS. Controversies in the evaluation and management of chronic cough. Am J Respir Crit Care Med 2011;183:708-715.

10. Chang AB, Lasserson TJ, Kiljander TO, Connor FL, Gaffney JT, Garske LA. Systematic review and meta-analysis 
of randomised controlled trials of gastro-oesophageal reflux interventions for chronic cough associated with gastro-oesophageal reflux. BMJ 2006;332:11-17.

11. Tanaka S, Hirata K, Kurihara N, Yoshikawa J, Takeda T. Effect of loratadine, an $\mathrm{H}_{1}$ antihistamine, on induced cough in non-asthmatic patients with chronic cough. Thorax 1996;51:810-814.

12. Pizzichini MM, Pizzichini E, Parameswaran K, et al. Nonasthmatic chronic cough: no effect of treatment with an inhaled corticosteroid in patients without sputum eosinophilia. Can Respir J 1999;6:323-330.

13. Ponsioen BP, Hop WC, Vermue NA, Dekhuijzen PN, Bohnen AM. Efficacy of fluticasone on cough: a randomised controlled trial. Eur Respir J 2005;25:147-152.

14. Chaudhuri R, McMahon AD, Thomson LJ, et al. Effect of inhaled corticosteroids on symptom severity and sputum mediator levels in chronic persistent cough. J Allergy Clin Immunol 2004;113:1063-1070.

15. Lee SY, Cho JY, Shim JJ, et al. Airway inflammation as an assessment of chronic nonproductive cough. Chest 2001;120:1114-1120.

16. Boulet LP, Milot J, Boutet M, St Georges F, Laviolette M. Airway inflammation in nonasthmatic subjects with chronic cough. Am J Respir Crit Care Med 1994;149:482489.

17. Chai H, Farr RS, Froehlich LA, et al. Standardization of bronchial inhalation challenge procedures. J Allergy Clin Immunol 1975;56:323-327.

18. Pin I, Gibson PG, Kolendowicz R, et al. Use of induced sputum cell counts to investigate airway inflammation in asthma. Thorax 1992;47:25-29.

19. Cox G, Whitehead L, Dolovich M, Jordana M, Gauldie J, Newhouse MT. A randomized controlled trial on the effect of inhaled corticosteroids on airways inflammation in adult cigarette smokers. Chest 1999;115:1271-1277.

20. Everett CF, Kastelik JA, Thompson RH, Morice AH. Chron- ic persistent cough in the community: a questionnaire survey. Cough 2007;3:5.

21. Gibson P, Wang G, McGarvey L, et al. Treatment of unexplained chronic cough: CHEST Guideline and Expert Panel Report. Chest 2016;149:27-44.

22. Pavord ID, Chung KF. Management of chronic cough. Lancet 2008;371:1375-1384.

23. Bolser DC. Older-generation antihistamines and cough due to upper airway cough syndrome (UACS): efficacy and mechanism. Lung 2008;186 Suppl 1:S74-S77.

24. Poe RH, Harder RV, Israel RH, Kallay MC. Chronic persistent cough. Experience in diagnosis and outcome using an anatomic diagnostic protocol. Chest 1989;95:723-728.

25. Curley FJ, Irwin RS, Pratter MR, et al. Cough and the common cold. Am Rev Respir Dis 1988;138:305-311.

26. Ribeiro M, Pereira CA, Nery LE, Beppu OS, Silva CO. High-dose inhaled beclomethasone treatment in patients with chronic cough: a randomized placebo-controlled study. Ann Allergy Asthma Immunol 2007;99:61-68.

27. Han B, Jang SH, Kim YJ, et al. The efficacy of inhaled corticosteroid on chronic idiopathic cough. Tuberc Respir Dis 2009;67:422-429.

28. Bardin PG, Van Heerden BB, Joubert JR. Absence of pulmonary aspiration of sinus contents in patients with asthma and sinusitis. J Allergy Clin Immunol 1990;86:8288.

29. Millqvist E. TRP channels and temperature in airway disease-clinical significance. Temperature (Austin) 2015;2:172177.

30. Khalid S, Murdoch R, Newlands A, et al. Transient receptor potential vanilloid 1 (TRPV1) antagonism in patients with refractory chronic cough: a double-blind randomized controlled trial. J Allergy Clin Immunol 2014;134:56-62.

31. Antonicelli L, Micucci C, Bonifazi F. Is ethanol-induced bronchospasm an inflammation-driven event? Allergy 2006;61:270-271. 